\title{
Embolización venosa transcatéter de várices gástricas con coils
}

\section{Transcatheter venous coil embolization of gastric varices}

\author{
Oscar Rivero Rapalino, ${ }^{1}$ Camilo Barragán Leal, ${ }^{2}$ Diego Salcedo Miranda, ${ }^{3 *}$ Laura Quintero Rojas. ${ }^{3}$
}

\footnotetext{
1 Radiólogo Intervencionista, Hospital Universitario Fundación Santa Fe, Universidad El Bosque, Bogotá, Colombia.

2 Radiólogo, Hospital Universitario Fundación Santa $\mathrm{Fe}$, Universidad El Bosque, Bogotá, Colombia.

3 Residente de Cirugía General, Hospital Universitario Fundación Santa Fe, Universidad El Bosque, Bogotá, Colombia.
}

*Correspondencia: dsalcedo_ub@hotmail.com

Fecha recibido: $11 / 05 / 18$

Fecha aceptado: 29/06/18

\begin{abstract}
Resumen
Los espirales de embolización (coils o microcoils) son los agentes más comúnmente usados, con un éxito técnico reportado en aproximadamente el $81-100 \%$ de los casos. Los espirales o coils se encuentran disponibles en una amplia variedad de configuraciones y tamaños, lo que permite acomodarse a vasos de distintos calibres; además, por su buena radiopacidad, tienen la ventaja de ser liberados con precisión. Los espirales o coils son el análogo de una ligadura arterial quirúrgica, debido a que producen una oclusión mecánica por su posicionamiento en el lumen vascular, lo que disminuye el flujo sanguíneo, y sus fibras sintéticas tienen un efecto trombogénico adicional. Caso: presentamos cuatro casos del Hospital Universitario Fundación Santa Fe de pacientes con várices gástricas tratadas satisfactoriamente con embolización transcatéter con coils, los cuales tuvieron un buen resultado técnico; además presentamos una revisión de la literatura.
\end{abstract}

\section{Palabras clave \\ Sangrado digestivo, várices gástricas, embolizacion, coils.}

\begin{abstract} the literature.

\section{Keywords}

Digestive bleeding, gastric varices, embolization, coils.
\end{abstract}

Coils and microcoils, the most commonly used embolization agents, have reported technical success rates ranging from $81 \%$ to $100 \%$ of cases. The spirals or coils are available in a wide variety of configurations and sizes which fit into vessels of different calibers. They have good radiopacity allowing for accurate release. Coils are the analogue of a surgical arterial ligation, because they produce mechanical occlusion due to their positioning in the vascular lumen. This decreases blood flow while their synthetic fibers have an additional thrombogenic effect. Case: We present four cases of coil embolization treatment of gastric varicose veins at our institution. All procedures were successful and had good technical results. We also present a review of

\section{INTRODUCCIÓN}

La embolización es la terapia endovascular de elección en el tratamiento de la hemorragia aguda del tracto gastrointestinal superior, incluyendo un origen hepatobiliar y del tracto digestivo inferior. Generalmente se requiere una cateterización arterial supraselectiva o una cateterización venosa utilizando un microcatéter con un calibre menor a 3 French (F), el cual es avanzado de forma coaxial a través de un catéter diagnóstico, usualmente de $5 \mathrm{~F}$, hacia aquellos segmentos vasculares distales o más próximos al sitio de sangrado. Esta técnica permite una embolización más selectiva, y evita otros territorios vasculares, lo cual disminuye el riesgo de isquemia intestinal $(1,2)$. 
En la hemorragia digestiva alta, la embolización arterial es factible, ya que no conlleva un riesgo isquémico significativo asociado, debido a la amplia red de colaterales que irrigan el tracto gastrointestinal superior. La embolización debe realizarse en el segmento arterial proximal y distal al foco hemorrágico con el objetivo de prevenir un nuevo episodio de sangrado, al evitar la irrigación retrógrada de la arteria involucrada; esto es conocido como oclusión de la puerta de entrada y de la puerta de salida del sitio de sangrado (2).

En algunos casos, los episodios de hemorragia digestiva son intermitentes y no se logran identificar durante la arteriografía; sin embargo, teniendo en cuenta la rica irrigación vascular del tracto gastrointestinal superior es posible realizar una embolización empírica o "ciega" de los vasos que suplen el segmento donde probablemente se encuentra la lesión que origina el sangrado. Se ha reportado que este tipo de embolización empírica puede ser realizada hasta en el $46 \%$ de los casos, y se ha demostrado que no hay una diferencia estadísticamente significativa en el resultado clínico de estos pacientes (2-4). Incluso es posible una embolización de ramas de la arteria hepática sin un riesgo isquémico significativo debido a la irrigación dual del hígado ( $75 \%$ por la vena porta y $25 \%$ por la arteria hepática) (2).

Las várices gástricas también han sido manejadas desde hace 25 años con obliteración retrógrada transvenosa con balón (balloon-occluded retrograde transvenous obliteration, BRTO), la cual requiere un agente esclerosante y un balón permanente para poder retener el agente esclerosante en la várice gástrica, y para optimizar la esclerosis de los vasos generando trombosis precoz local e impidiendo que esta se vaya a la circulación sistémica. Se han descrito numerosas variables a esta técnica que no requieren la oclusión con balón para reemplazarlo con dispositivos embólicos, entre los cuales encontramos una amplia variedad de materiales de embolización; sin embargo, no hay una evidencia conclusiva sobre cuál es el mejor agente de embolización en comparación con los otros. En la práctica, su uso depende de la experiencia y preferencia del operador y de la disponibilidad del producto. Los agentes más comúnmente utilizados son los espirales o coils, las esponjas de gelatina absorbible $\left(\right.$ Gelfoam $\left.^{\circledast}\right)$, las partículas y los agentes embolizantes líquidos $(2,5)$.

Los espirales de embolización (coils o microcoils) son los agentes más comúnmente usados, con un éxito técnico reportado en aproximadamente el 81-100 \% de los casos. Los espirales o coils se encuentran disponibles en una amplia variedad de configuraciones y tamaños, lo que permite acomodarse a vasos de distintos calibres, y por su buena radiopacidad, tienen la ventaja de ser liberados con precisión. Los espirales o coils son el análogo de una ligadura arterial quirúrgica, debido a que producen una oclusión mecánica por su posicionamiento en el lumen vascular, lo que disminuye el flujo sanguíneo, y sus fibras sintéticas tienen un efecto trombogénico adicional $(1,5,6)$.

Las esponjas de Gelfoam $^{\circledast}$ y las partículas de polivinil alcohol o de otros materiales (gelatina trisacryl) se han utilizado exitosamente; sin embargo, el control de su liberación es difícil y menos precisa en comparación con los coils. Otra desventaja de las partículas de embolización es que pueden alcanzar la circulación distal intramural intestinal con un mayor riesgo de isquemia, y pueden comprometer la circulación de un territorio vascular no deseado; por lo tanto, se deben usar las partículas de mayor tamaño, por encima de 500 micras $(\mu)$. Las esponjas de Gelfoam ${ }^{\circledR}$ producen una oclusión temporal, con la teórica ventaja de permitir la recanalización de los vasos embolizados entre 2-6 semanas; no obstante, la isquemia intestinal se presenta en las primeras 8-12 horas luego de la interrupción del flujo sanguíneo, por lo tanto no tienen el beneficio adicional de evitar este riesgo $(1,5,7)$.

El éxito técnico de la embolización es cercano al $100 \%$,y puede controlar satisfactoriamente la hemorragia digestiva en el $80-90 \%$ de los pacientes. El sangrado recurrente es infrecuente, excepto en los casos de angiodisplasias, malformaciones arteriovenosas o lesiones inflamatorias, y se ha reportado en aproximadamente el $15 \%$ de los pacientes. Los nuevos episodios de sangrado pueden requerir una nueva arteriografía y embolización, sin aumentar el riego de isquemia intestinal $(1,5,8,9)$.

Las mayores complicaciones relacionadas con la embolización arterial incluyen la isquemia intestinal y la nefropatía inducida por el medio de contraste. Con el desarrollo de la tecnología del microcatéter y con el perfeccionamiento de los materiales de embolización, las complicaciones isquémicas clínicamente relevantes ocurren en menos del $2 \%$ de los pacientes. La mayoría de las complicaciones son insignificantes y no requieren un tratamiento adicional $(2,5)$.

Adicionalmente, las várices gástricas presentan ciertos aspectos patológicos y hemodinámicos un poco diferentes con respecto a las várices esofágicas. La mayoría de las várices gástricas son secundarias a una hipertensión portal. Otras causas incluyen las várices secundarias a una trombosis de la vena esplénica. La BRTO es otro procedimiento altamente efectivo en el tratamiento mínimamente invasivo de las várices gástricas aisladas, especialmente en aquellos pacientes con pobre reserva hepática que no son candidatos a una TIPS. Esta técnica utiliza un catéter con un balón distal que obstruye el flujo sanguíneo durante la inyección del esclerosante a través de las venas de la comunicación portosistémica (generalmente gastrorenal), y permite el adecuado llenado de las várices con el tiempo suficiente para que la escleroterapia sea efectiva, mientras previene el reflujo de la sustancia esclerosante hacia la circulación sistémica o portal (2); sin embargo, hemos logrado sustituir el 
balón por coils con unos buenos resultados y un buen control del sangrado. Presentamos así la serie de cuatro casos de control de hemorragia gastrointestinal alta debido a várices gástricas con shunt gastrorrenal en nuestra institución, manejada con embolización transcatéter venosa con coils, la cual obtuvo un buen resultado técnico y un buen control del sangrado en los cuatro pacientes.

\section{SERIE DE CASOS}

\section{Primer caso}

Paciente masculino de 74 años con antecedente de hepatocarcinoma metastásico y cirrosis, con invasión tumoral de la vena porta, quien consulta por un cuadro de dos días de deposiciones melénicas. Se realiza una endoscopia de vías digestivas altas (EVDA) en la que se encuentran várices esofágicas pequeñas sin estigmas de sangrado, una várice de aproximadamente $15 \mathrm{~mm}$ con signos rojos a nivel subcardial hacia el fondo gástrico y una zona ulcerada en la superficie sin sangrado activo. Se considera que el paciente tiene sangrado digestivo alto portal hipertensivo secundario a una várice fúndica grande, y no es candidato a manejo endoscópico, por lo cual se solicita una valoración por radiología intervencionista; allí le toman una tomografía de abdomen en la que se hayan várices tortuosas adyacentes a la curvatura menor del estómago (Figura 1A). Se realiza una venografía selectiva opacificando las várices tortuosas en una curvatura menor, con algunas venas de drenaje paraespinales, un shunt gastrorrenal (Figura 1B) y una embolización con coils (Figura 1C) ocluyendo el flujo en várices.
El procedimiento se realizó sin complicaciones, por lo que se dio egreso a los dos días.

\section{Segundo caso}

Paciente masculino de 60 años con antecedente de cirugía de Puestow por neoplasia pancreática, quien asiste por melenas y anemia con hemoglobina de $7 \mathrm{~g} / \mathrm{dL}$. En la EVDA se observa sangre en la cavidad gástrica y un coagulo grande que no se remueve en la pared posterior de curvatura mayor del cuerpo proximal, de donde se observa sangrado en capa debajo de este; se realiza escleroterapia con solución de adrenalina y se realiza un control de esta. Además, se realiza una EVDA de control en la que se encuentran dos paquetes variciales grandes sin sangrado activo en el fondo del estómago, pero por el tamaño no es posible realizar una ligadura de estos, por lo que se requiere embolización. Se realiza una tomografía de abdomen en la que se encuentran várices tortuosas en curvatura menor del estómago (Figura 2A). Se realiza una venografía selectiva opacificando las várices tortuosas en una curvatura menor, un shunt gastrorrenal (Figura 2B), y una embolización con coils (Figura 2C) ocluyendo el flujo en las várices. El procedimiento se realizó sin complicaciones, y se dio de alta al día siguiente.

\section{Tercer caso}

Paciente femenina de 66 años con antecedente de cirrosis biliar primaria e hipertensión portal, quien consulta por dos episodios de hematemesis. En la EVDA se encontraron 4
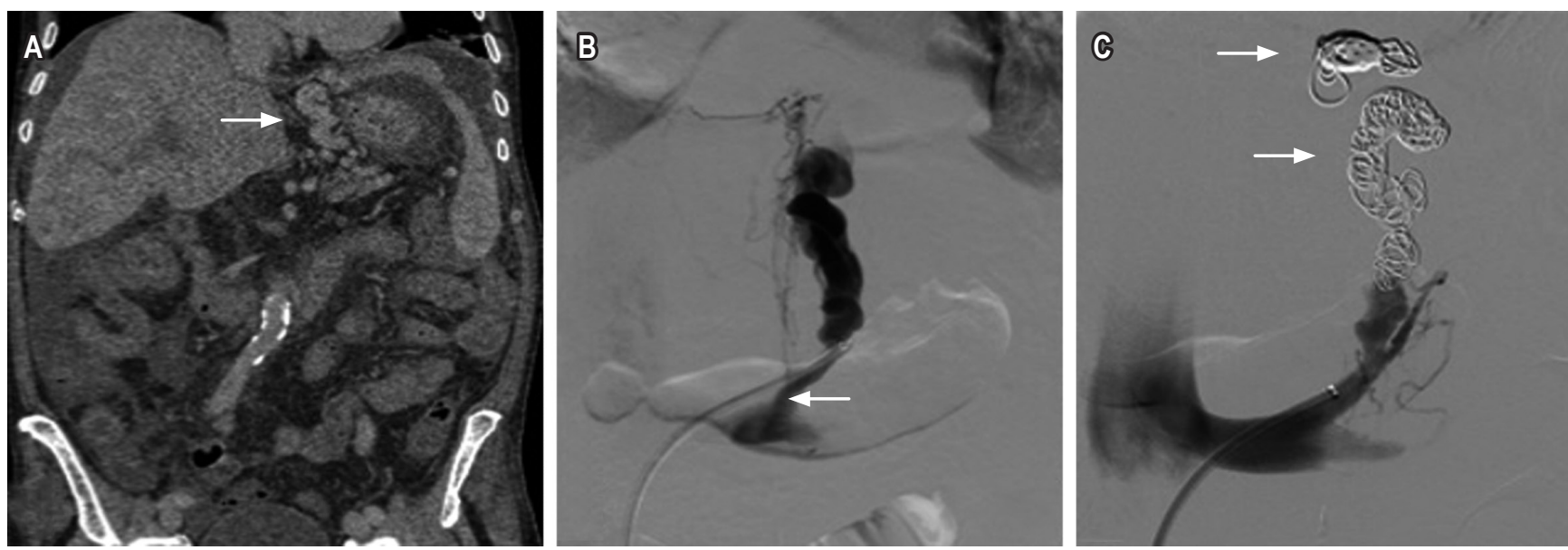

Figura 1. Hombre de 74 años. Cirrosis, várices gástricas con shunt gastrorrenal. A. Tomografía abdominal contrastada, corte coronal con ventana a tejidos blandos que muestra várices tortuosas adyacentes a la curvatura menor del estómago (flecha). B. Venografía selectiva opacificando várices tortuosas en curvatura menor, con algunas venas drenaje paraespinales y shunt gastrorrenal (flecha). C. Embolización con coils (flecha) ocluyendo el flujo en várices. 

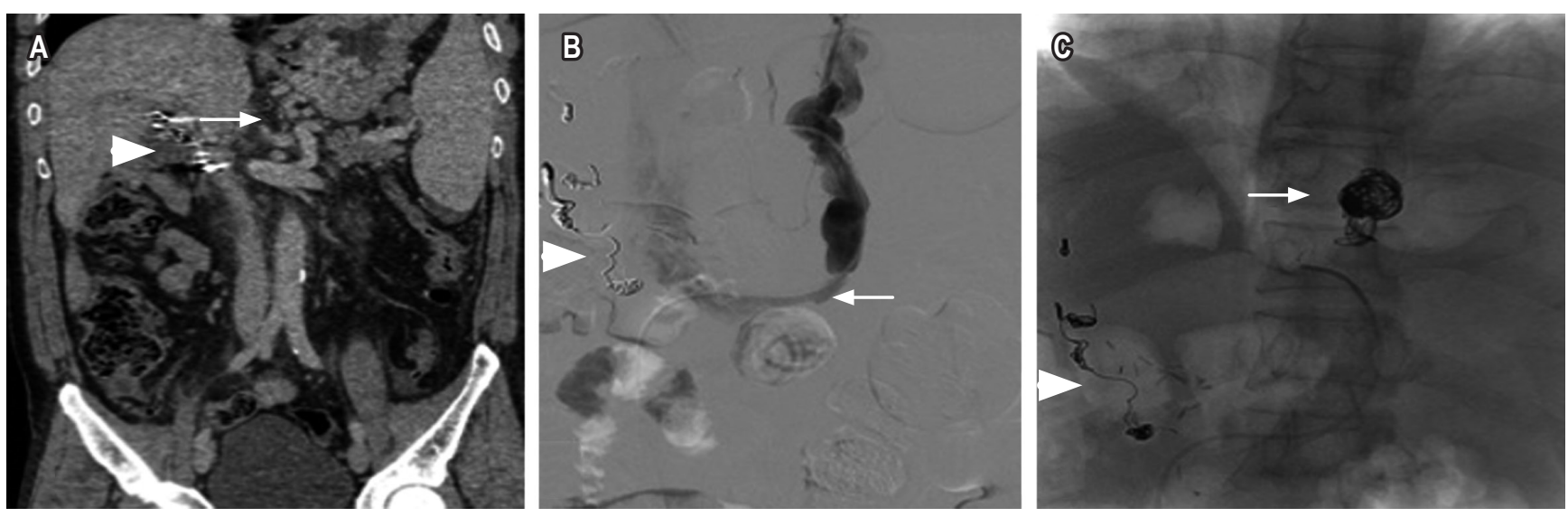

Figura 2. Hombre de 60 años, antecedente de pancreatoduodenectomía, quien desarrolló pseudoaneurisma de arteria hepática, embolizado con coils (triángulo A, B y C) con várices gástricas y shunt gastrorrenal. A. Tomografía abdominal contrastada, corte coronal con ventana a tejidos blandos que muestra várices tortuosas en una curvatura menor del estómago (flecha). B. Venografía selectiva opacificando las várices tortuosas en una curvatura menor y shunt gastrorrenal (flecha). C. Embolización con coils (flecha) ocluyendo el flujo en várices.

cordones varicosos dilatados en el tercio distal del esófago, que ocupan el $25 \%$ de la luz esofágica, sin signos rojos, ni estigmas de sangrado agudo reciente o antiguo. En el lago gástrico, se encontraron restos hemáticos antiguos. No se encontró evidencia de sangrado activo, y a nivel subcardial hacia la curvatura mayor se encontró una várice grande sin estigmas de sangrado agudo, reciente o antiguo. Se realiza una resonancia magnética de abdomen en la que se encuentran várices tortuosas adyacentes a la curvatura menor del estómago y shunt gastrorrenal (Figura 3A); debido a estos hallazgos, se considera que la paciente tiene un riesgo alto de resangrado, por lo que se considera que requiere embolización. Se realiza una venografía selectiva opacificando la várice tortuosa en una curvatura menor, shunt gastrorrenal (Figura 3B) y embolización con coils (Figura 3C) ocluyendo el flujo en várices. Este procedimiento fue realizado sin complicaciones y se dio alta temprana a la paciente.

\section{Cuarto caso}

Paciente femenina de 69 años con antecedente de cirrosis hepática de origen alcohólico, con puntaje Child Pugh C,
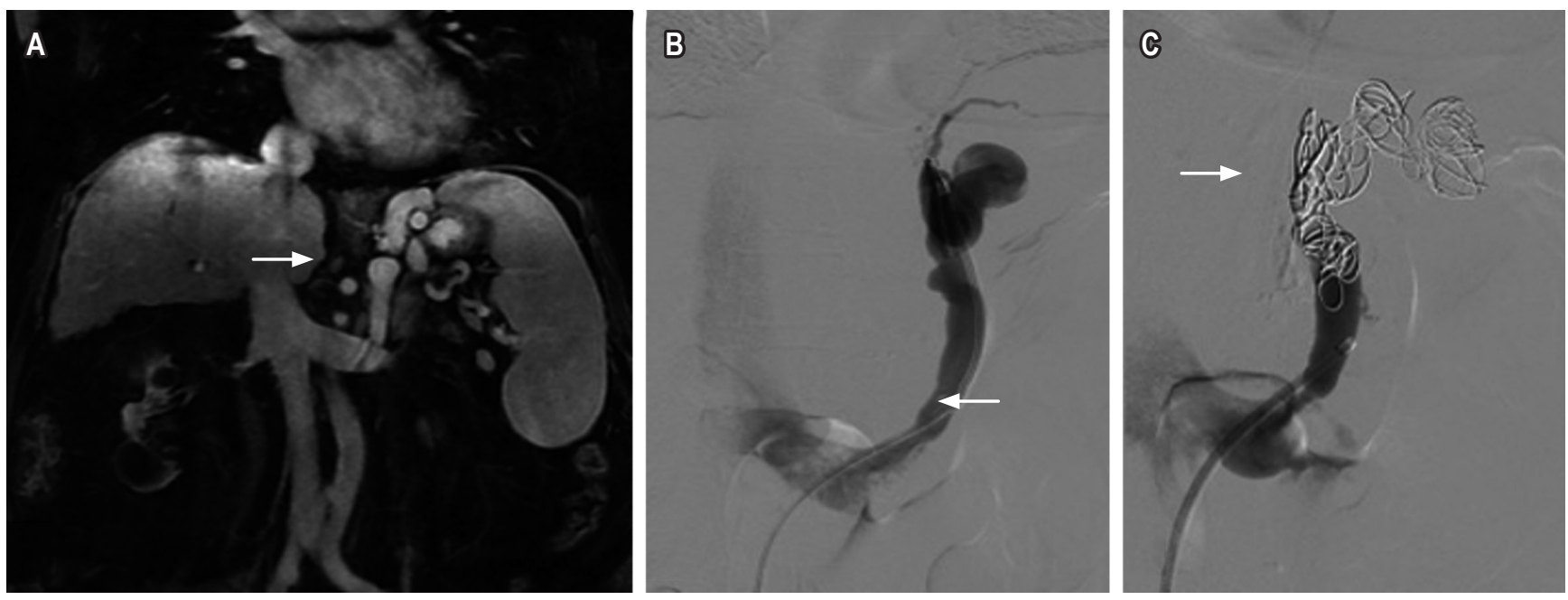

Figura 3. Mujer de 66 años con cirrosis hepática. A. Resonancia magnética abdominal contrastada, corte coronal T1 con saturación grasa, várices tortuosas adyacentes a la curvatura menor del estómago y shunt gastrorrenal (flecha). B. Venografía selectiva opacificando várice tortuosa en una curvatura menor y shunt gastrorrenal (flecha). C. Embolización con coils (flecha) ocluyendo el flujo en várices. 
quien consulta por un sangrado digestivo alto de difícil manejo endoscópico de origen variceal esofágico y gástrico. El paciente ameritó protección de la vía aérea debido al riesgo de broncoaspiración, múltiples transfusiones y soporte vasopresor. Se considera la realización de una embolización por vía transparietohepática. En la tomografía de abdomen contrastada se observan várices tortuosas adyacentes a la curvatura menor del estómago (Figura 4A). Se realiza una venografía selectiva de acceso retrógrado portal opacificando várices tortuosas en la curvatura menor gástrica (Figura 4B) y una embolización con coils y Gelfoam ${ }^{\circledast}$ (Figura 4C) ocluyendo el flujo en várices. El procedimiento se realizó sin complicaciones y se pudo controlar el sangrado de la paciente.

\section{DISCUSIÓN}

El sangrado digestivo superior secundario a várices gastroesofágicas es una complicación mayor en pacientes con cirrosis e hipertensión portal, con una incidencia reportada entre el 4-14 \%, que cada vez se presenta con mayor frecuencia debido al incremento de la patología hepática, la cual produce, a su vez, el aumento de la tensión en la vena porta, donde la cirrosis es la causa más frecuente $(10,11)$. En el grupo de pacientes con cirrosis que se presentan con un sangrado digestivo superior, las várices son la causa de aproximadamente el $59 \%$ de los casos, seguidas por las úlceras pépticas en el $16 \%$ de los casos $(12,13)$.

El sistema de várices gástricas se define como el shunt gastrorrenal, las várices gástricas (submucosa y extragástrica) y los conductos venosos aferentes dominantes portales. Entre el 60-80 \% de las várices gástricas se asocian a una comunicación portosistémica espontánea en el lado izquierdo, incluyendo, principalmente, una derivación gastrorrenal o gastroesplénica (14); estos se han relacionado con una mayor mortalidad, y su tratamiento endoscópico es menos efectivo $(12,14)$, por ello la terapia con embolización y el control angiográfico de la hemorragia gastrointestinal por várices gástricas es la indicación de manejo. Este subgrupo de pacientes requiere una intervención angiográfica que permita localizar el origen del sangrado y su tratamiento por vía endovascular $(2,5,15,16)$. Por esta razón, se realizó una embolización transcatéter venosa con coils de los 4 pacientes.

La técnica actual de embolización con coils en el tratamiento de la hemorragia gastrointestinal aguda controla con éxito el sangrado en aproximadamente el 80-90\% de los pacientes $(8,9)$, ya que para el tratamiento de la hemorragia gastrointestinal aguda es una técnica segura basada en la fisiología de que las fibras del coil provocan una trombosis del vaso, mientras que la propia coil funciona como una andamio para el trombo, con eventos adversos importantes que ocurren en menos del $2 \%$ de los pacientes (2). Sin embargo, una desventaja de este mecanismo es que imposibilita el acceso endosvascular futuro a la lesión específica, pero las tasas de nuevas hemorragias después de la embolización con coil están entre 0-40 \% (6). Una parte de los pacientes embolizados, de manera muy selectiva, desarrollarán cambios isquémicos menores, asintomáticos y autolimitados, como úlceras pequeñas. Además, es poco probable que una embolización con coil muy selectiva produzca un retraso en el infarto, ya que esta complicación ocurre típicamente casi de inmediato (es decir, dentro de las primeras 48 horas) después del procedimiento de
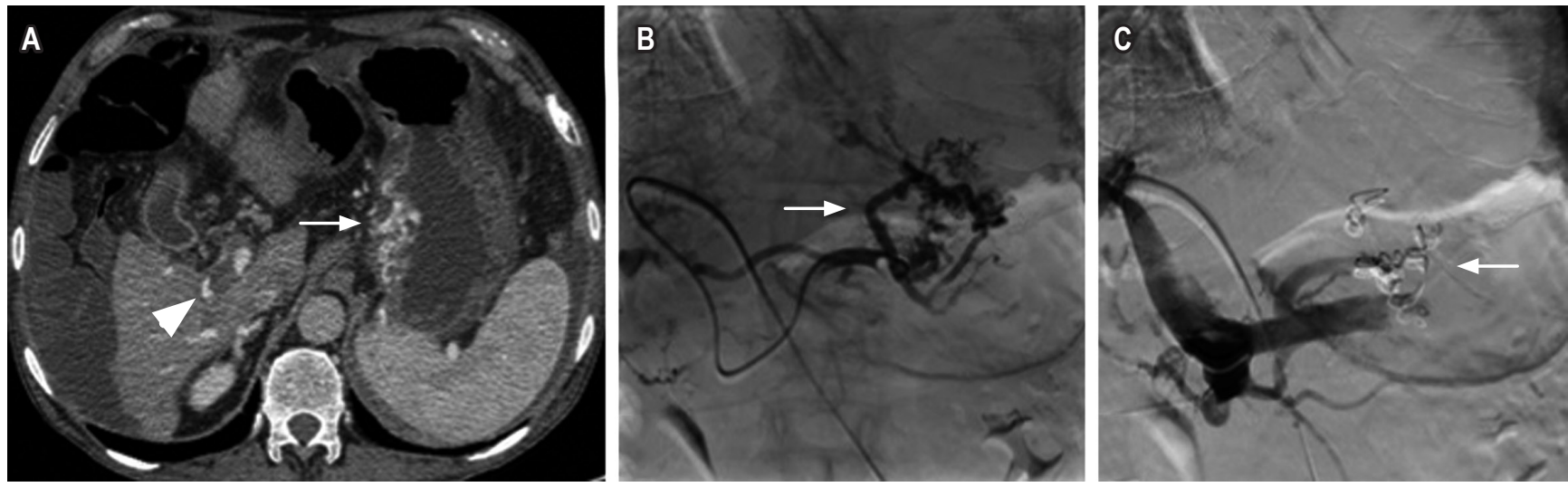

Figura 4. Mujer de 66 años con cirrosis hepática no candidata para TIPS por trombosis de la porta (triángulo A). A. Tomografía abdominal contrastada, corte axial con ventana a tejidos blandos, várices tortuosas adyacentes a la curvatura menor del estómago (flecha). B. Venografía selectiva de acceso retrógrado portal opacificando várices tortuosas en la curvatura menor gástrica (flecha). C. Embolización con coils (flecha) ocluyendo el flujo en várices. 
embolización. La embolización no objetiva con coils es poco frecuente, ya que las coils se introducen solo después de que se haya ingresado con éxito un microcatéter en el vaso diana. Por esta razón, uno debe elegir con cuidado el tamaño adecuado de los coils, ya que un coil que está sobredimensionado con respecto al vaso objetivo puede desplazar al microcatéter de su posición supraselectiva. Esto podría llevar al despliegue del coil en una ubicación no objetiva. Del mismo modo, los coils de tamaño insuficiente pueden no ocluir adecuadamente el vaso objetivo o pueden alojarse distalmente a la lesión que se va a tratar, disminuyendo las tasas de éxito del procedimiento (5). Aunque algunos autores como Ford y colaboradores prefieren sobredimensionar los coils por al menos $15-20 \%$ en el sistema venoso para minimizar la probabilidad de migración debido al gran calibre de la vena gástrica izquierda y las várices gástricas combinado con el alto flujo a través de la derivación esplenorrenal (17). En nuestros casos no se presentaron complicaciones mayores ni menores durante ni después a la embolización venosa transcatéter con coils, y no se presentaron migraciones posteriores de los coils.

La BRTO es un procedimiento altamente efectivo en el tratamiento mínimamente invasivo de las várices gástricas aisladas, especialmente en aquellos pacientes con pobre reserva hepática que no son candidatos a TIPS, como algunos de nuestros pacientes. Esta técnica utiliza un catéter con un balón distal que obstruye el flujo sanguíneo durante la inyección del esclerosante a través de las venas de la comunicación portosistémica (generalmente gastrorenal), y permite el llenado adecuado de las várices con el tiempo suficiente para que la escleroterapia sea efectiva, mientras previene el reflujo de la sustancia esclerosante hacia la circulación sistémica o portal (2); sin embargo, consideramos, como en algunas revisiones de la literatura, que la embolizacion transcatéter venosa con coils ha tendido a reemplazar a la BRTO, debido a que es un procedimiento con una carga logística que puede desgastar al equipo de radiología intervencionista, aumentar el gasto de los recursos hospitalarios; además, sustituir el balón por coils es técnicamente factible (18), por ello, nuestra técnica utilizada es la embolización venosa transcatéter con coils, con la cual, hasta el momento, hemos obtenido buenos resultados.

\section{CONCLUSIÓN}

La embolización venosa transcatéter con coils es una alternativa efectiva y segura a la cirugía de emergencia en pacientes con patología hepatobiliar, en quienes un procedimiento quirúrgico podría aumentar la morbimortalidad considerablemente. Los avances técnicos y la disponibilidad cada vez mayor de angiografía diagnóstica y de embolización transcatéter han fortalecido esta opción de trata- miento del sangrado de origen variceal gástrico, refractaria a la terapia médica y endoscópica.

\section{Aprobación ética}

Se obtuvo el consentimiento informado por escrito del paciente para la publicación de este documento.

\section{Fuente de financiación}

Este artículo no contó ni requirió apoyo financiero alguno.

\section{REFERENCIAS}

1. Navuluri R, Kang L, Patel J, Van Ha T. Acute lower gastrointestinal bleeding. Semin Intervent Radiol. 2012 Sep;29(3):178-86. doi: 10.1055/s-0032-1326926.

2. Navuluri R, Patel J, Kang L. Role of interventional radiology in the emergent management of acute upper gastrointestinal bleeding. Semin Intervent Radiol. 2012 Sep;29(3):169-77. doi: $10.1055 / \mathrm{s}-0032-1326925$.

3. Aina R, Oliva VL, Therasse E, Perreault P, Bui BT, Dufresne $\mathrm{MP}$, et al. Arterial embolotherapy for upper gastrointestinal hemorrhage: outcome assessment. J Vasc Interv Radiol. 2001 Feb;12(2):195-200. doi: 10.1016/S10510443(07)61825-9.

4. Padia SA, Geisinger MA, Newman JS, Pierce G, Obuchowski NA, Sands MJ. Effectiveness of coil embolization in angiographically detectable versus non-detectable sources of upper gastrointestinal hemorrhage. J Vasc Interv Radiol. 2009 Apr;20(4):461-6. doi: 10.1016/j.jvir.2009.01.006.

5. Walker TG, Salazar GM, Waltman AC. Angiographic evaluation and management of acute gastrointestinal hemorrhage. World J Gastroenterol. 2012 Mar 21;18(11):1191-201. doi: 10.3748/wjg.v18.i11.1191.

6. d'Othée BJ, Surapaneni P, Rabkin D, Nasser I, Clouse M. Microcoil embolization for acute lower gastrointestinal bleeding. Cardiovasc Intervent Radiol. 2006 JanFeb;29(1):49-58. doi: 10.1007/s00270-004-0301-4.

7. Abdel-Aal AK, Bag AK, Saddekni S, Hamed MF, Ahmed FY. Endovascular management of nonvariceal upper gastrointestinal hemorrhage. Eur J Gastroenterol Hepatol. 2013 Jul;25(7):755-63. doi: 10.1097/MEG.0b013e32835fb9a9.

8. Patel TH, Cordts PR, Abcarian P, Sawyer MA. Will transcatheter embolotherapy replace surgery in the treatment of gastrointestinal bleeding? (2)(2). Curr Surg. 2001 May;58(3):323327. doi: 10.1016/S0149-7944(01)00417-2.

9. Schenker MP, Duszak R Jr, Soulen MC, Smith KP, Baum RA, Cope C, et al. Upper gastrointestinal hemorrhage and transcatheter embolotherapy: clinical and technical factors impacting success and survival. J Vasc Interv Radiol. 2001 Nov;12(11):1263-71. doi: 10.1016/S10510443(07)61549-8.

10. Sarin SK, Lahoti D, Saxena SP, Murthy NS, Makwana UK. Prevalence, classification and natural history of gastric vari- 
ces: a long-term follow-up study in 568 portal hypertension patients. Hepatology. 1992 Dec;16(6):1343-9. doi: 10.1002/hep.1840160607.

11. Castillo O, Palacios F, Yoza M, Contardo C, Soriano CC. Uso de Cianoacrilato en la Terapia Endoscópica de várices Gástricas: Experiencia en el Hospital Nacional Edgardo Rebagliati Martins del 2006 al 2010. Rev Gastroenterol Perú. 2011;31(3):208-215.

12. Gibson JA, Odze RD. Pathology of diseases that cause upper gastrointestinal tract bleeding. Gastrointest Endosc Clin N Am. 2011 Oct;21(4):583-96. doi: 10.1016/j. giec.2011.07.006.

13. Sostres C, Lanas A. Epidemiology and demographics of upper gastrointestinal bleeding: prevalence, incidence, and mortality. Gastrointest Endosc Clin N Am. 2011 Oct;21(4):567-81. doi: 10.1016/j.giec.2011.07.004.

14. Saad WE. Endovascular management of gastric varices. Clin Liver Dis. 2014 Nov;18(4):829-51. doi: 10.1016/j. cld.2014.07.005.
15. Wee E. Management of nonvariceal upper gastrointestinal bleeding. J Postgrad Med. 2011 Apr-Jun;57(2):161-7. doi: 10.4103/0022-3859.81868.

16. Edelman DA, Sugawa C. Lower gastrointestinal bleeding: a review. Surg Endosc. 2007 Apr;21(4):514-20. doi: 10.1007/ s00464-006-9191-7.

17. Ford JM, Shah H, Stecker MS, Namyslowski J. Embolization of large gastric varices using vena cava filter and coils. Cardiovasc Intervent Radiol. 2004 Jul-Aug;27(4):366-9. doi: $10.1007 /$ s00270-004-0071-z.

18. Saad WE, Nicholson DB. Optimizing logistics for balloonoccluded retrograde transvenous obliteration (BRTO) of gastric varices by doing away with the indwelling balloon: concept and techniques. Tech Vasc Interv Radiol. 2013 Jun;16(2):152-7. doi: 10.1053/j.tvir.2013.02.006. 